\title{
Reliability of the NEO Five Factor Inventory Short Form for assessing personality after stroke
}

\section{Running header: Reliability of the NEO-FFI after stroke}

Toni Dwan ${ }^{1}$, Professor Tamara Ownsworth ${ }^{1}$, Dr Caroline Donovan ${ }^{1}$, Dr Ada Ho Yan Lo ${ }^{2}$

${ }^{1}$ School of Applied Psychology and Menzies Health Institute Queensland, Griffith University, Brisbane, Australia

${ }^{2}$ Princess Alexandra Hospital, Brisbane, Australia

Contact for correspondence:

Toni Dwan

School of Applied Psychology

Griffith University

Mt Gravatt Campus

Mt Gravatt, 4122

Australia

Ph: $\quad+61737353305$

Fax: $\quad+61737353388$

Email: t.dwan@griffith.edu.au

Word count: 6000

Number of tables: 5 


\begin{abstract}
Background: It is well recognised that an individual's personality characteristics influence their psychological adjustment after stroke. However, there is a lack of research on the reliability of personality inventories for stroke. This study primarily aimed to evaluate the reliability of the NEO-Five Factor Inventory (NEO-FFI) for assessing pre-morbid personality and personality changes after stroke. Further aims were to investigate changes in personality during the hospital-to-home transition period and examine associations between personality and mood.
\end{abstract}

Methods: Forty participants with stroke (52.5\% male, $M$ age $=65.55$ years) were recruited at time of hospital discharge and completed the NEO-FFI, Centre for Epidemiologic Studies Depression and Geriatric Anxiety Inventory. Significant others completed an Informant version of the NEO-FFI. Stroke participants were re-assessed on the NEO-FFI at 1-month and 4-months post-discharge. Forty matched controls also completed the NEO-FFI. Results: Internal consistency was adequate for the NEO-FFI $(\alpha=.57-.86)$, although low for agreeableness. There was fair to excellent concordance between self-rated and informant versions of the NEO-FFI (ICC $=.58-.78)$. Significant positive associations were found between neuroticism and mood ( $r=.50-.68)$, and significant negative associations were found between extraversion and mood ( $r=-.33-.36)$ and agreeableness and anxiety $(r=-.43)$. Self-ratings of stroke participants on the NEO-FFI at discharge did not significantly differ from matched controls. Extraversion levels significantly decreased, and agreeableness levels significantly increased between discharge and 1 and 4-months post-discharge.

Conclusions: Overall, the results support the reliability of the NEO-FFI for assessing personality characteristics in the context of stroke.

Keywords: Stroke, personality, NEO-FFI, depression, anxiety. 


\section{Reliability of the NEO Five Factor Inventory Short Form for assessing personality after}

\section{stroke}

Stroke is a leading cause of death and disability worldwide and most commonly occurs in people aged over 65 (Thrift et al., 2014). Stroke is associated with diverse physical, cognitive and behavioural impairments that affect every day functioning and quality of life. The prognostic significance of neurological factors such as the severity, location and type of stroke is well established for particular outcomes such as survival and functional status; however, such indices are not reliably related to mental health outcomes.

High rates of depression and anxiety have typically been reported following stroke (29-31\%; Broomfield et al., 2014; Hackett and Pickles, 2014). The development of depression is associated with poorer cognitive functioning (Robinson and Jorge, 2016), reduced adherence and response to rehabilitation (Ahn et al., 2015), higher levels of disability (Ayerbe et al., 2013), and lower quality of life for individuals with stroke and their caregivers (Godwin et al., 2013). A Cochrane review found a lack of evidence supporting the efficacy of psychological and pharmacological interventions for treating post-stroke depression and anxiety (Hackett et al., 2008). Nonetheless, there is some evidence that early psychological interventions focusing on promoting well-being and adjustment after stroke can be effective in preventing the development of depression (Hackett et al., 2008). To support these early intervention efforts there is a need to identify the characteristics that place individuals at greatest risk of developing depression or anxiety.

It is well recognised that the nature and severity of stroke can only partly account for differences in psychological well-being (van Mierlo et al., 2014). When people experience a serious illness later in life they draw upon many resources, including their own longstanding patterns of coping. Individuals’ premorbid personality, or characteristic thinking styles, emotions, and behaviour, is likely to have a major influence on how they adjust to changes in 
their functioning and lifestyle after stroke. A wealth of research on individual differences highlights how personality characteristics influence health behaviours and psychological well-being (e.g., Villieux et al., 2016).

Accordingly, there is a growing body of research investigating the influence of personality on post-stroke adjustment. A systematic review by van Mierlo et al. (2014) on the influence of psychological factors on health-related quality of life after stroke identified that “problems of temperament and personality functions” were moderately and negatively associated with health-related quality of life $(r=0.26-0.49)$. Many studies assessing personality after stroke have employed the NEO Personality Inventory-Revised (NEO-PI-R; 240 items; Costa and McCrae, 1992) or the short form, NEO Five Factor Inventory (NEOFFI; 60 items; Costa and McCrae, 1992). Both inventories assess the following five major domains of personality (i.e., “Big Five”): Neuroticism, Extraversion, Openness, Agreeableness and Conscientiousness (Costa and McCrae, 1992).

The NEO has been used in several stroke studies that examined the impact of stroke on personality and/or associations between personality and mood (e.g., Greenop et al., 2009; Kim et al., 2013; Storor and Byrne, 2006). In a meta-analysis pooling the findings of four cohort studies, Jokela and colleagues (2014) found that stroke was associated with greater personality change on the NEO than the onset of other chronic conditions, such as cancer, diabetes and arthritis. There is also evidence that personality characteristics influence psychological adjustment following stroke (see review by van Mierlo et al., 2014). In particular, people high in premorbid neuroticism have been found to be more likely to experience depression, anxiety and irritability after stroke (e.g., Kim et al., 2013). Further, lower levels of agreeableness were found to be associated with increased depression at two weeks post-stroke (Storor and Byrne, 2006) and greater agitation/aggression and irritability at three months post-stroke (Greenop et al., 2009). Conversely, Kim and colleagues (2013) 
found that higher levels of extroversion, agreeableness, and conscientiousness early after stroke were associated with better quality of life at 3-months follow-up post-stroke. Interestingly, those higher on openness to experience were more likely to report significant improvement on QoL measures over the three-month period (Kim et al., 2013). The importance of understanding the role of personality in post-stroke adjustment is further highlighted by research demonstrating a link between personality, depression and mortality (Morris et al., 1993).

Studies in the stroke literature have typically assessed personality at a single time point; for example, at 2-4 weeks post-stroke (e.g., Hwang et al., 2011) or at two or three months post-stroke (e.g., Kim et al., 2013). The approach of assessing personality early after stroke assumes that reports at that time reflect individuals’ premorbid personality. To directly test this assumption it would be necessary to measure personality characteristics prior to and after stroke. Such prospective research requires pre-identification of people at risk of stroke which is rarely feasible. An alternative approach is to compare the early retrospective ratings of premorbid personality in people with stroke to healthy controls matched on demographic characteristics typically related to personality (see Goldberg et al., 1998). A similar approach was employed by Tate (2003) to investigate pre-injury personality characteristics and personality change following traumatic brain injury. An assessment of personality characteristics at several months post-stroke makes it difficult to determine the extent to which ratings reflect premorbid characteristics, post-stroke personality changes, or a combination of these (Kim et al., 2013; Nijsse et al., 2015). More generally, self-reports of personality are potentially influenced by the direct functional effects of stroke (e.g., cognitive and language deficits), emotional factors (e.g., trauma reactions related to a life threatening event and stroke-related disability) and contextual factors such as the hospital environment. To date, however, there has been no longitudinal study of personality changes after stroke. 
Due to concerns about various factors affecting the reliability of self-ratings some studies have used informants to rate the premorbid personality of the person with stroke (e.g., Hwang et al., 2011). An informant's perspective may differ from that of the person with stroke for many reasons, including the level of familiarity in their relationship, the informant's own psychological state, and the somewhat subjective nature of personality characteristics in general. Given these issues, there is a need for research to examine both internal consistency and concordance between self and informant ratings of personality in the context of stroke.

Administration of personality measures during hospitalisation may help to identify individuals most at risk of developing psychological distress prior to and following discharge. The NEO-FFI short form is considered particularly useful given the tool's well-established psychometric properties in the general population and the need to reduce assessment burden (e.g., Costa and McCrae, 1992). In circumstances in which the person with stroke is unable to complete the measure (e.g., due to language impairment or severe cognitive impairment), it may be advantageous to use an informant version to inform psychological management plans.

In summary, there is a lack of research on the reliability of the NEO-FFI for assessing pre-morbid personality and personality change after stroke. No study to date has investigated changes in personality using a longitudinal design. Accordingly, to understand the reliability of the NEO-FFI for assessing personality characteristics in the context of stroke, the primary and novel aims of the present study were to:

1) Examine the internal consistency of self-report and informant-report versions of the NEO-FFI as well as the concordance between self and informant ratings.

2) Compare the personality characteristics of individuals recently hospitalised for stroke and healthy controls matched on age, gender and education. 
3) To utilise a longitudinal design to examine changes in personality during the hospital-to-home transition period after stroke (i.e., at time of discharge, 1-month post-discharge and 4-months post-discharge).

For the purposes of replicating previous research, a secondary objective of the study was to investigate associations between self-ratings on the NEO-FFI and measures of depression and anxiety at the time of hospital discharge. Guided by the findings of previous stroke and personality research (Afshar et al., 2015; Hwang et al., 2011; Kim et al., 2013), it was hypothesised that neuroticism would be positively associated with depression and anxiety, and that extraversion and agreeableness would be negatively associated with depression and anxiety. The comparison of personality characteristics between stroke participants and matched controls, and the longitudinal investigation of personality change, represented exploratory components.

\section{Method}

\section{Participants}

Individuals with stroke were recruited from the geriatric rehabilitation and assessment unit at a major metropolitan hospital. Patients were screened by their treating clinical psychologist or neuropsychologist according to the following eligibility criteria: diagnosis of stroke and medically stable, adequate receptive and expressive communication skills in English (note: this was confirmed using the Speech Questionnaire; Lincoln, 1982), adequate cognitive capacity to provide informed consent, and absence of a neurodegenerative, psychotic or substance abuse disorder. A total of 67 potential participants were screened for inclusion in the study. Of these, 19 did not meet inclusion criteria due to moderate to severe aphasia $(n=9)$, suspected neurodegenerative disorder $(n=4)$, substance abuse disorder $(n=$ 
3) or a medically unstable condition ( $n=3)$, and eight were unable to provide consent or did not provide consent. A total of 40 eligible patients consented to participate.

Participants included 21 males (52.5\%) and 19 females (47.5\%) with an age range of 37-93 years $(M=65.55, S D=14.13)$ and mean time since stroke of 36.7 days $(S D=22.27)$. Education level for the sample ranged from 5 to 24 years $(M=11.51, S D=4.34)$. Medical reports indicated that most participants sustained an ischemic stroke (68\%) located in the right hemisphere (50\%). Functional independence, as assessed by the Modified Barthel Index (MBI), varied from “Severe dependence” to "Independence”, with a mean score in the “Moderate independence” range $(M=84.00, S D=23.04)$. Participants’ global cognitive status on the Repeatable Battery for the Assessment of Neuropsychological Status (RBANS) spanned the "Extremely low" to "Superior" range, with a mean score in the "Low average" range $(M=83.90, S D=15.65)$. Table 1 presents the demographic and clinical characteristics of each participant with stroke.

\section{[Insert Table 1]}

All stroke participants were asked to nominate a close relative or long-term friend to complete the informant version of the NEO-FFI. Of the 40 participants, 22 had a close relative or friend available. Informants included spouses (72.7\%), adult children (22.7\%) and one close family friend (4.6\%).

A convenience sample of control participants was recruited through the researchers' social networks. To optimise matching, 62 adults with a demographic profile similar to the stroke sample were screened for matching purposes. Participants were included in the study if they were aged 36 - 93 years, spoke English fluently, and had no history of a neurological event or other major medical conditions. Following the screening and matching process, the final control sample included 40 participants (45\% male) aged 36-88 years $(M=63.05, S D=$ 12.84), with an education level of $7-17$ years $(M=11.40, S D=2.55)$. A chi-square test and 
independent $t$-tests confirmed that the stroke and control samples did not significantly differ on gender $\left(\chi^{2}=0.45, p=.50\right)$, age $(t=-0.83, p=.51)$ or education $(t=-0.14, p=.81)$. The participant groups in the current study are summarised in Figure 1.

[Insert Figure 1]

\section{Materials}

\section{Personality}

Neuroticism, Extroversion, Openness to Experience - Five Factor Inventory (NEOFFI; Costa and McCrae, 1992). The NEO-FFI consists of 60 items rated on a 5-point scale (Strongly Disagree to Strongly Agree), and is designed to measure the five broad personality domains of Neuroticism, Extraversion, Openness to experience, Agreeableness and Conscientiousness. The NEO-FFI has been validated against the NEO-PI and corresponding scales, with correlations ranging from .75 (Conscientiousness) to .89 (Neuroticism). Additionally, correlations between the informant rating Form R of the NEO-FFI scales and self-reports have ranged from .47 (Extraversion) to .68 (agreeableness) $(p<.001)$, suggesting adequate concordance between self- and observer ratings (Foltz et al., 1997). Convergent, construct and criterion validity have been demonstrated in many studies using the NEO-FFI, with convergent validity correlations ranging from .56 to .62, and no correlation exceeding .20 for divergent validity (Costa and McCrae, 1992). In a large validation study ( $n=1539)$, Costa and McCrae (1992) reported Cronbach’s alpha coefficients ranging from .68 (Agreeableness) to 89 (Neuroticism). The latent structure of the NEO-FFI was found to be consistent with its scoring key, thus supporting construct validity. Both the NEO-PI and NEO-FFI have been used in research on psychological adjustment to stroke, traumatic brain injury and other neurological disorders (e.g., Greenop et al., 2009). 


\section{Mood}

Centre for Epidemiologic Studies Depression (CES-D; Radloff, 1977). The CES-D is a self-report measure of depression that consists of 20 items. Participants rate on a four-point scale (rarely $=0$; most of the time $=3$ ) how often they felt a particular way over the last week. Example items include: 'I was bothered by things that usually don't bother me' and 'I felt that I could not shake off the blues even with help from my family or friends'. Scores of 16 or greater are in the clinical range for depression. The CES-D has been the focus of rigorous psychometric testing and has been found to have good internal consistency ( $\alpha>.85$; Hann et al., 1999). In the stroke population, scores on the CES-D have been found to be highly correlated with other depression measures $(r=.57-.82, p<.005)$.

Geriatric Anxiety Inventory (GAI; Pachana et al., 2007). The GAI is a measure of anxiety that was specifically designed for use in the older adult population. It consists of 20 questions that are endorsed 'yes' or 'no'. 'Yes' answers are summed to yield a total score, with scores of 10 or greater indicating clinical levels of anxiety. The GAI has been found to have excellent internal consistency with an alpha of .91 among normal older adults and .93 in a clinical sample of older people. Excellent test-retest reliability has also been demonstrated $(r=.91)$, as well as good concurrent validity $(.70-.80)$ with other anxiety measures (Pachana et al., 2007).

\section{Procedure}

Ethics approval for the study was gained from both hospital and university human ethical review committees. Following informed consent procedures and assessment of cognitive (RBANS) and physical status (MBI), participants in the stroke sample were individually administered the personality and mood questionnaires in the following order: NEO-FFI, CES-D and GAI. The assessment at time of discharge was conducted face-to-face at the hospital in a private assessment room, or at home shortly after discharge, in a quiet 
room with no distractions. Participants with stroke and their informants were asked to complete the NEO-FFI according to the typical characteristics of the person with stroke, prior to the stroke. The follow-up assessments conducted at 1-month post-discharge and 4-months post-discharge were conducted by telephone or face-to-face during a home visit as preferred by the participant. During the follow-up assessments, participants were asked to complete the NEO-FFI according to their current ways of thinking, feeling and behaving.

Control participants were recruited from the researchers' social networks using a snowball convenience sampling approach. Potential participants were sent an email with a link to an online survey or had the option of receiving hardcopies of questionnaires in the mail with reply paid envelopes. Completed surveys were scored and entered into the Statistical Package for Social Sciences (22) for analysis.

\section{Results}

\section{Data analysis}

The data were screened for missing values and checked for relevant assumptions in accordance with Tabachnick and Fidell (2013). There were no missing data for any personality or mood variables. For the reliability analyses, Cronbach's alpha was used to examine internal consistency and the intraclass correlation coefficient (ICC) was used to assess concordance between self and informant ratings on the NEO-FFI. The relevant assumptions for parametric tests (i.e., absence of outliers, independence, normality, sphericity and homogeneity of variance) were met; thus, independent-sample $t$-tests were conducted to compare personality ratings between the stroke participants (time of discharge) and the healthy control group. One-way repeated measures ANOVA was used to examine changes in personality between time of discharge and 1-month and 4-months post-discharge, with a Bonferroni adjustment applied for multiple comparisons $(p<.017)$. Inspection of descriptive data and scatterplots indicated that assumptions regarding absence of outliers, normality, 
linearity and homoscedasticity were met; therefore, Pearson's product moment correlation was used to examine associations between personality domains and mood at time of discharge. Confidence intervals (95\% CI) are reported for all analyses.

\section{Internal consistency and concordance}

Internal consistency for the GAI and CES-D in the current stroke sample was excellent $(\alpha=.93 ; C I=.90 \sim .96)$ and good $(\alpha=.88 ; C I=.83 \sim .93)$ respectively. As shown in Table 2, internal consistency for the personality domains of the NEO-FFI in the stroke sample varied between poor $(\alpha=.57 ; C I=.34 \sim .74)$ for agreeableness and $\operatorname{good}(\alpha=.86 ; C I$ $=.79 \sim .92)$ for extraversion. On the informant version, internal consistency also ranged from poor $(\alpha=.66 ; C I=.41 \sim .85$, openness $)$ to $\operatorname{good}(\alpha=.87 ; C I=.77 \sim .94$, conscientiousness $)$. Similarly, for the control group, the internal consistency of self-ratings varied between poor $(\alpha=.68 ; C I=.51 \sim .81$, conscientiousness $)$ and good (.83; $C I=.73 \sim .90$, neuroticism). These findings are broadly consistent with the alpha coefficients $(\alpha=.68-.89)$ reported for the NEOFFI validation sample by Costa and McCrae (1992).

\section{[Insert Table 2]}

Interclass correlation coefficients between self-report (stroke group) and informant versions of the NEO-FFI personality domains ranged between fair $(I C C=.58 ; C I=.09 \sim .83)$ for agreeableness and excellent $(I C C=.78 ; C I=.46 \sim .91)$ for neuroticism (see Table 3). Such findings indicate that the concordance between self- and informant reports on the NEO-FFI was generally satisfactory. Nonetheless, caution is needed when interpreting the results for domains with low reliability and large confidence intervals.

[Insert Table 3] 


\section{Comparison between stroke and control samples on the NEO-FFI}

Independent-sample $t$-tests were conducted to compare personality characteristics of individuals recently hospitalised for stroke (i.e., premorbid personality ratings) and healthy controls matched on age, gender and education. There were no significant differences between the groups on any personality domain (see Table 5).

\section{A longitudinal examination of personality change on the NEO-FFI}

As shown in Figure 1, 27 of the 40 participants with stroke assessed at time of discharge were available for the follow-up assessments at both 1-month and 4-months postdischarge (note: 31 participants were retained in the study at 1-month post-discharge, however, 4 of these participants were not available at 4-months post-discharge). Of the 13 participants who were not available for both follow-up assessments, three could not be contacted, three had only consented to the initial assessment, three declined to continue, two were too unwell to continue due to deterioration in their health status, and two had died. An attrition analysis was conducted to compare stroke participants who were retained in the study ( $n=27)$ with those not retained at both follow-up assessments $(n=13)$ on demographic and stroke characteristics (i.e., age, gender, education, stroke type, cognitive status, functional independence) and personality and mood variables. There was only one significant difference between the groups, which was found on the personality dimension of openness. Participants retained in the study at follow-up had significantly lower ratings of openness ( $M$ $=43.52, S D=8.04)$ than participants not retained $(M=50.70, S D=10.30, t[38]=-2.41, p=$ $.02)$.

One-way repeated measures ANOVA were conducted on self-ratings on the NEOFFI. A significant change over time was found for the personality domains of extraversion (Pillai's Trace $=.26, F[2,25]=4.37, p=.02)$ and agreeableness (Pillai's Trace $=.26, F[2$, $25]=4.29, p=.03)$. Post-hoc tests with a Bonferroni adjustment identified a significant 
decrease in extraversion between time of discharge $(M=49.00, S D=13.25)$ and 1 -month post-discharge $(M=44.56, S D=12.05 ; t[26]=2.94, p=.007, C I=1.33 \sim 7.56)$. There was also a significant decrease in extraversion scores between time of discharge and 4-months post-discharge $(M=43.63, S D=11.43 ; t[26]=-2.81, p=.009, C I=-9.30 \sim-1.44)$. There was no significant difference in extraversion levels between 1-month post-discharge and 4-months post-discharge $(t[26]=.85, p=.40)$.

For the personality domain of agreeableness, post-hoc tests indicated a significant increase in scores between time of discharge $(M=47.26, S D=9.81)$ and 1-month postdischarge $(M=50.74, S D=8.42 ; t[26]=-2.86, p=.008, C I=-5.89 \sim-.98)$. There was also a significant increase in agreeableness between time of discharge and 4-months post-discharge $(M=50.93, S D=9.33 ; t[26]=2.63, p=.014, C I=.80 \sim 6.54)$. There was no significant difference in agreeableness between 1-month post-discharge and 4-months post-discharge ( $t$ $[26]=-.18, p=.86)$.

\section{[Insert Table 4]}

\section{Associations between personality domains and mood}

At time of discharge, approximately $28 \%$ of stroke participants were in the clinical range $(\geq 16)$ for depression on the CESD $(M=12.65, S D=10.03)$. A slightly lower proportion of the sample $(22.5 \%)$ was in the clinical range $(\geq 10)$ for anxiety on the GAI $(M=$ $5.23, S D=5.70)$. As shown in Table 4, significant positive associations were found between levels of neuroticism and depression $(r=.50, p<.01 ; C I=.22 \sim .70)$ and anxiety $(r=.68$, $p<.001 ; C I=.47 \sim .82)$. Significant negative associations were found between extraversion and depression $(r=-.36, p<.05 ; C I=-.6 \sim-.05)$ and anxiety $(r=-.33, p<.05 ; C I=-.58 \sim-.02)$, and between agreeableness and anxiety $(r=-.43, p<.01 ; C I=-.65 \sim-.14)$. There were no other significant associations between the NEO-FFI personality dimensions and mood. 


\section{Discussion}

This study primarily aimed to evaluate the reliability of the NEO-Five Factor Inventory (NEO-FFI) for assessing pre-morbid personality and personality changes after stroke. Overall, there were some mixed findings concerning the reliability of the NEO-FFI. Specifically, internal consistency was poor $(\alpha<.70)$ for the agreeableness and openness scales, but fair to good for the other three scales. However, concordance between self-rated and informant versions of the NEO-FFI was satisfactory for all personality domains with coefficients ranging from fair to excellent. In relation to the second aim, there were no significant differences on the NEO-FFI between the matched control group and stroke sample at time of discharge. With respect to the third aim, there was evidence of post-stroke personality changes whereby levels of extraversion significantly decreased and levels of agreeableness significantly increased between discharge and the 1 and 4-months postdischarge assessments. In terms of the secondary objective, as hypothesised, significant positive associations were found between neuroticism and depression and anxiety; whereas negative associations were found between extraversion and depression and anxiety. Further, agreeableness was significantly and negatively associated with anxiety but not depression.

The mixed findings concerning internal consistency of the NEO-FFI $(\alpha=.57-.86)$ for the stroke group were similar to the pattern reported for the large validation sample (Costa and McCrae, 1992), in which alphas ranged from .68 (Agreeableness) to .89 (Neuroticism). Coefficients for both agreeableness and openness were lower than desirable $(<.70)$ for the stroke participants' self-rated version, and internal consistency was also low for openness on the informant version $(\alpha=.66)$. Although the results are broadly consistent with the NEOFFI validation sample, it is important to consider what may have contributed to the particularly low reliability of the agreeableness domain of the NEO-FFI in this study. In 
terms of assessment context, participants had recently experienced a stroke and were administered the personality inventory in hospital or within days of being discharged home.

Agreeableness is considered to be a superordinate personality trait that encompasses facets such as trust, straightforwardness, altruism, compliance, modesty, and sympathy (Costa and McCrae, 1992). The facets underlying this personality construct may be more situationally influenced and affected by recent illness and hospitalisation than other NEO domains. Consistent with this view, in a review of 51 studies that used the NEO scales (NEO PI, NEO PI-R and NEO-FFI) Caruso (2000) found that internal consistency was lowest for the domain of agreeableness, and was particularly low for studies involving clinical samples $(M \alpha=.62)$, including hospitalised patients. Caruso (2000) proposed that the domain of agreeableness as measured by the NEO scales may reflect state rather than trait characteristics. It is noteworthy that internal consistency for agreeableness was fair $(\alpha=.75)$ and good $(\alpha=.81)$ for the informant and control groups respectively. The low reliability ( $\alpha=$ .57) for the stroke sample supports Caruso’s view that ratings on the agreeableness domain are sensitive to contextual influences (e.g., health status and environment), and thus may reflect more situational characteristics. As discussed later, these findings highlight the need for caution when interpreting the finding that levels of agreeableness increased following hospital discharge.

An examination of concordance between the self-rated and informant versions of the NEO revealed good (extraversion, openness and conscientiousness) to excellent (neuroticism) consistency for stroke participants and their relatives/close friends. Interestingly, concordance was somewhat lower for agreeableness (fair) and informants rated participants with stroke as slightly more agreeable than their own self-ratings. Overall, such findings indicate that relatives or close friends can reliably report the personality characteristics of individuals with stroke at the time of hospital discharge. This may be particularly useful in situations where 
the individual is unable to complete the NEO-FFI due to impaired language or cognitive capacity.

Self-ratings on the NEO-FFI were compared between the stroke sample at discharge and matched controls. The lack of significant between-group differences on any personality dimension indicates that early ratings on the NEO-FFI after stroke are consistent with healthy adults with similar demographic characteristics. Such findings contrast with those of Jokela et al. (2014) who found that individuals at longer time points post-stroke reported lower extraversion, conscientiousness, agreeableness and openness, and increased neuroticism than controls. Although it cannot be concluded that early ratings of personality after stroke represent premorbid personality, these ratings provide a meaningful baseline for examining changes in personality over time.

The within-group analyses identified that levels of extraversion significantly decreased for stroke participants between the discharge assessment and both follow-up assessments (1 and 4-months post-discharge). Such findings are consistent with previous research which indicates that extraversion declines following the onset of chronic illness. In a pooled analysis of four cohort studies which involved seven chronic illnesses including stroke (heart disease, stroke, diabetes, cancer, respiratory disease, arthritis, and hypertension), Jokela et al. (2014) found a significant decrease in extroversion relative to controls. The decrease in extraversion was attributed to fatigue and other physical symptoms which lower people’s energy and positivity and reduce their desire for social contact or enjoyment of the same. Given that stroke is associated with physical, sensory-perceptual, cognitive and communication impairments, it is not surprising that participants reported themselves as less outgoing, talkative and socially-oriented than prior to their stroke. Further research is needed to understand the stroke-related impairments contributing to these personality changes and to determine whether such changes persist more long-term after stroke. 
In contrast to the findings of Jokela et al. (2014), a significant increase was found in levels of agreeableness of stroke participants between discharge and the two follow-up assessments. Such findings indicate that participants perceived themselves as more trusting, cooperative and willing to get along with others than prior to their stroke, which may be related to their greater reliance or dependence on others for support in the first few months post-stroke. However, due to the low internal consistency of the agreeableness domain for the current sample, which suggests that the scale may not reflect a unidimensional construct, caution is needed when interpreting changes in scores on this domain. Further research is needed to understand what the items on this domain reflect (i.e., state or trait characteristics) in the context of stroke and other chronic illnesses (see Caruso, 2000).

Consistent with previous findings in the stroke literature (Greenop et al., 2009; Hwang et al., 2011), participants with higher neuroticism reported increased levels of depression and anxiety. In the general literature it is well established that people high in neuroticism experience more negative affect and labile mood and have less effective coping mechanisms than people low in neuroticism, which in turn increases their vulnerability to psychological disorders (Costa and McCrae, 1992). In contrast to the findings for neuroticism, stroke participants with higher extraversion reported lower levels of depression and anxiety. People high in extraversion are typically sociable, assertive, active and energetic (Costa and McCrae, 1992). They usually have strong social ties and multiple group memberships which may offer social support during difficult times (Roohafza et al., 2016). Greater social networks and maintenance of group memberships has been found to buffer the effects of stroke on psychological well-being (Haslam et al., 2008). Further, high agreeableness was associated with lower anxiety in the present sample. This is consistent with the findings Afshar et al. (2015) who reported that low agreeableness was related to high anxiety in a community-based sample of 4763 adults. One of the key facets of agreeableness 
is trust. Low levels of trust have been found to contribute to negative affect including anxiety (Glinski and Page, 2012).

Some further methodological limitations of the current study are important to acknowledge. Firstly, the sample size was lower than optimal for examining reliability and this may have contributed to the low alpha values. For reliability analyses a sample size of 30-49 is considered "fair", whereas a sample of $\geq 50$ is considered "good” (Terwee et al., 2012). The modest sample size also contributed to large confidence intervals for particular personality domains (i.e., agreeableness, conscientiousness and openness); hence, the findings may provide less precise estimates of reliability for some domains than others. It is recommended that future studies on reliability endeavour to recruit a minimum sample of 50 participants. Further, it was not possible to recruit participants on a consecutive basis into the study due to staffing changes in the unit. The attrition analysis identified that participants retained in the study were lower on openness than those lost to follow-up, which was unexpected given that higher openness to experience is associated with intellectual curiosity (Costa and McCrae, 1992). More generally, due to the convenience sample and attrition, there is a need for caution in generalising the findings to the broader stroke population. A prospective cohort study that assesses personality characteristics early after stroke and examines changes across multiple time points is recommended. In particular, the long-term effects of stroke on levels of extraversion and predictors of changes in this personality domain are important to understand given that extraversion is positively associated with health behaviours and psychological well-being (Villieux et al., 2016).

\section{Conclusions}

There is growing recognition that personality characteristics help to account for differences in psychological well-being following stroke (van Mierlo et al., 2014). This study found that the NEO-FFI generally had sound internal consistency and there was good 
concordance between self-rated and informant versions. Higher levels of neuroticism and lower levels of extraversion and agreeableness were associated with greater mood symptoms. Ratings on the NEO-FFI at time of discharge after stroke were found to be consistent with the ratings of healthy adults with similar demographic characteristics. Finally, there was some evidence of personality change after stroke, with a decrease in extraversion and an increase in agreeableness evident at 1 and 4-months post-discharge. These findings support the reliability of the NEO-FFI for identifying people at increased risk of poor psychological outcomes based on their pre-morbid personality (e.g., high neuroticism) or changes in longstanding traits (e.g., decreased extraversion) that affect their ability to effectively cope with the challenges posed by stroke.

\section{Conflict of Interest:}

None.

\section{Acknowledgements}

The first author (TD) would like to acknowledge the Australian Postgraduate Award Research Scholarship for financial support throughout candidature. The authors would also like to thank staff at the Royal Brisbane and Women's hospital for their assistance with design, screening and recruitment. In particular, we would like to thank Emmaline Falconer, Elizabeth Torbey and Rosemary Argall for their expertise and support during recruitment.

\section{Description of authors' roles}

All authors (TD, TO, CD \& AL) contributed intellectually to the study design and were involved in the drafting of the manuscript and/or critically revising the intellectual content. TD and TO had the lead role in preparing the manuscript, and conceived the study design, methodology and research procedures. AL facilitated participant recruitment. TD collected 
the data and conducted the statistical analyses. All authors read and approved the final manuscript. 


\section{References}

Afshar, H., Roohafza, H., Hassanzadeh-Keshteli, A., Sharbafchi, M. R., Feizi, A. and Adibi, P. (2015). Association of personality traits with psychological factors of depression, anxiety, and psychological distress: A community based study. International Journal of Body, Mind and Culture, 2, 105-114.

Ahn, D.-H., Lee, Y.-J., Jeong, J.-H., Kim, Y.-R. and Park, J.-B. (2015). The effect of poststroke depression on rehabilitation outcome and the impact of caregiver type as a factor of post-stroke depression. Ann Rehabil Med, 39, 74-80. doi: org/10.5535/arm.2015.39.1.74 Ayerbe, L., Ayis, S., Wolfe, C. D. A. and Rudd, A. G. (2013). Natural history, predictors and outcomes of depression after stroke: systematic review and meta-analysis. The British Journal of Psychiatry, 202, 14-21. doi:10.1192/bjp.bp.111.107664

Broomfield, N. M., Quinn, T. J., Abdul-Rahim, A. H., Walters, M. R. and Evans, J. J. (2014). Depression and anxiety symptoms post-stroke/TIA: prevalence and associations in cross-sectional data from a regional stroke registry. BMC Neurology, 14, 1-9. doi: 10.1186/s12883-014-0198-8

Caruso, J. C. (2000). Reliability generalization of the NEO personality scales. Educational and Psychological Measurement, 60, 236-254. doi: 10.1177/00131640021970484

Costa, P. T. and McCrae, R. R. (1992). Revised NEO personality inventory (NEO PI-R) and NEO five-factor inventory (NEO FFI): Professional manual. Odessa, Fl: Psychological Assessment Resources.

Foltz, C., Morse, J. Q., Calvo, N. and Barber, J. P. (1997). Self- and observer ratings on the NEO-FFI in couples: Initial evidence of the psychometric properties of an observer form. Assessment, 4, 287-295. doi:10.1177/107319119700400308 
Glinski, K. and Page, A. C. (2012). Modifiability of neuroticism, extraversion, and agreeableness by group cognitive behaviour therapy for social anxiety disorder. Behaviour Change, 27, 42-52. doi: http://dx.doi.org/10.1375/bech.27.1.42

Godwin, K. M., Ostwald, S. K., Cron, S. G. and Wasserman, J. (2013). Long-term health related quality of life of survivors of stroke and their spousal caregivers. The Journal of neuroscience nursing : journal of the American Association of Neuroscience Nurses, 45, 147154. doi:10.1097/JNN.0b013e31828a410b

Goldberg, L. R., Sweeney, D., Merenda, P. F. and Hughes, J. E. (1998). Demographic variables and personality: The effects of gender, age, education, and ethnic/racial status on self-descriptions of personality attributes. Personality and Individual Differences, 24, 393403. doi:10.1016/S0191-8869(97)00110-4

Greenop, K. R., Almeida, O. P., Hankey, G. J., van Bockxmeer, F. and Lautenschlager, N. T. (2009). Premorbid personality traits are associated with post-stroke behavioral and psychological symptoms: A three-month follow-up study in Perth, Western Australia. International Psychogeriatrics, 21, 1063-1071. doi:10.1017/S1041610209990457

Hackett, M. L., Anderson, C. S., House, A. O. and Xia, J. (2008). Interventions for treating depression after stroke. Cochrane Database of Systematic Reviews, 1 - 95. doi: 10.1002/14651858.CD003437.pub3

Hackett, M. L. and Pickles, K. (2014). Part I: frequency of depression after stroke: an updated systematic review and meta-analysis of observational studies. International Journal of Stroke, 9, 1017-1025. doi:10.1111/ijs.12357

Hann, D., Winter, K. and Jacobsen, P. (1999). Measurement of depressive symptoms in cancer patients: evaluation of the Center for Epidemiological Studies Depression Scale (CESD). Journal of psychosomatic research, 46, 437-443. doi:10.1016/S0022-3999(99)00004-5 
Hwang, S. I., Choi, K. I., Park, O. T., Park, S.-W., Choi, E. S. and Yi, S.-H. (2011). Correlations between pre-morbid personality and depression scales in stroke patients. Annals of Rehabilitation Medicine, 35, 328-336. doi:10.5535/arm.2011.35.3.328

Jokela, M., Hakulinen, C., Singh-Manoux, A. and Kivimäki, M. (2014). Personality change associated with chronic diseases: pooled analysis of four prospective cohort studies. Psychological Medicine, 44, 2629-2640. doi:10.1017/S0033291714000257

Kim, S., et al. (2013). Influences of personality traits on quality of life after stroke. European Neurology, 69, 185-192. doi:10.1159/000345699

Lincoln, N. B. (1982). The Speech Questionnaire: an assessment of functional language Ability. International Rehabilitation Medicine, 4, 114-117. doi:10.3109/09638288209166893 Morris, P. L. P., Robinson, R. G., \& Samuels, J. (1993). Depression, introversion and mortality following stroke. Australian and New Zealand Journal of Psychiatry, 27, 443-449. doi: 10.3109/00048679309075801

Nijsse, B., van Heugten, C. M., van Mierlo, M. L., Post, M. W. M., de Kort, P. L. M. and Visser-Meily, J. M. A. (2015). Psychological factors are associated with subjective cognitive complaints 2 months post-stroke. Neuropsychological Rehabilitation, 1-17. doi:10.1080/09602011.2015.1065280

Pachana, N. A., Byrne, G. J., Siddle, H., Koloski, N., Harley, E. and Arnold, E. (2007). Development and validation of the Geriatric Anxiety Inventory. International Psychogeriatrics, 19, 103-114. doi:10.1017/S1041610206003504

Radloff, L. S. (1977). The CES-D scale: A self-report depression scale for research in the general population. Applied Psychological Measurement, 1, 385-401. doi:10.1177/014662167700100306

Robinson, R. G. and Jorge, R. E. (2016). Post-stroke depression: A Review. American Journal of Psychiatry, 173, 221-231. doi:10.1176/appi.ajp.2015.15030363 
Storor, D. L. and Byrne, G. J. A. (2006). Pre-morbid personality and depression following stroke. International Psychogeriatrics, 18, 457-469.

Tabachnick, B. G. and Fidell, L. S. (2013). Using multivariate statistics. USA: Pearson Education.

Tate, R. L. (2003). Impact of pre-injury factors on outcome after severe traumatic brain injury: Does post-traumatic personality change represent an exacerbation of premorbid traits? Neuropsychological Rehabilitation, 13, 43-64. doi: 10.1080/09602010244000372

Tate, R. L. (2010). A compendium of tests, scales, and questionnaires: The practitioner's guide to measuring outcomes after acquired brain impairment. USA: Psychology Press. Terwee, C. B., Mokkink, L. B., Knol, D. L., Ostelo, R. W. J. G., Bouter, L. M. and de Vet, H. C. W. (2012). Rating the methodological quality in systematic reviews of studies on measurement properties: a scoring system for the COSMIN checklist. Quality of Life Research, 21, 651-657. doi: 10.1007/s11136-011-9960-1

Thrift, A. G., et al. (2014). Global stroke statistics. International Journal of Stroke, 9, 6-18. doi: 10.1111/ijs.12245

van Mierlo, M. L., Schröder, C., van Heugten, C. M., Post, M. W. M., de Kort, P. L. M. and Visser-Meily, J. M. A. (2014). The influence of psychological factors on health-related quality of life after stroke: a systematic review. International Journal of Stroke, 9, 341-348. doi: 10.1111/ijs.12149

Villieux, A., Sovet, L., Jung, S.-C. and Guilbert, L. (2016). Psychological flourishing: Validation of the French version of the Flourishing Scale and exploration of its relationships with personality traits. Personality and Individual differences, 88, 1-5. doi:10.1016/j.paid.2015.08.027 


\begin{tabular}{|c|}
\hline Control \\
Participants \\
$n=40$ \\
\hline
\end{tabular}

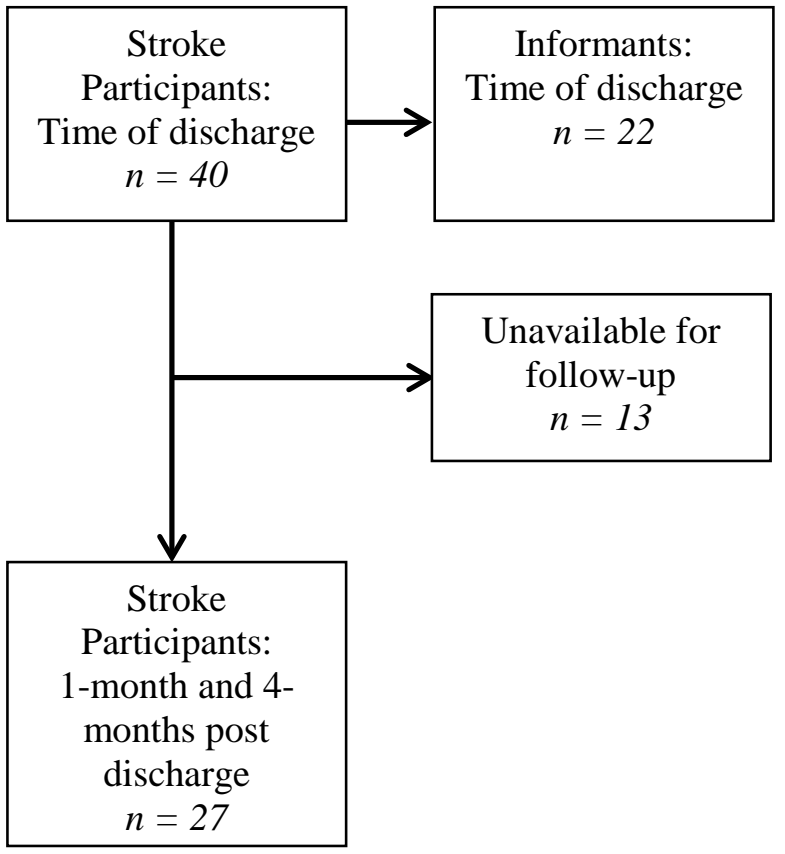

Figure 1. Participants in the current study 
Table 1

Characteristics of Stroke Participants $(n=40)$

\begin{tabular}{|c|c|c|c|c|c|}
\hline Gender & Age & Stroke Type & $\begin{array}{l}\text { Stroke } \\
\text { Location }\end{array}$ & $\begin{array}{l}\text { Global } \\
\text { RBANS } \\
\text { Score }\end{array}$ & MBI \\
\hline $\mathrm{F}$ & 79 & Haemorrhagic & Right & 52 & 100 \\
\hline $\mathrm{F}$ & 71 & Ischemic & Right & 77 & 30 \\
\hline M & 93 & Ischemic & Left & 79 & 100 \\
\hline M & 50 & Haemorrhagic & Left & 101 & 65 \\
\hline M & 84 & Haemorrhagic & Bilateral & 102 & 100 \\
\hline $\mathrm{F}$ & 65 & Haemorrhagic & Right & 106 & 100 \\
\hline M & 59 & Ischemic & Left & 90 & 100 \\
\hline M & 47 & Ischemic & Bilateral & 99 & 100 \\
\hline M & 55 & Ischemic & Right & 104 & 95 \\
\hline $\mathrm{F}$ & 41 & Ischemic & Right & 88 & 85 \\
\hline M & 85 & Ischemic & Left & 67 & 90 \\
\hline $\mathrm{F}$ & 76 & Ischemic & Bilateral & 57 & 100 \\
\hline M & 80 & Ischemic & Right & 100 & 100 \\
\hline M & 37 & Ischemic & Bilateral & 62 & 95 \\
\hline M & 55 & Ischemic & Right & 68 & 65 \\
\hline $\mathrm{F}$ & 61 & Haemorrhagic & Left & 77 & 100 \\
\hline M & 77 & Ischemic & Left & 73 & 95 \\
\hline M & 63 & Haemorrhagic & Right & 87 & 90 \\
\hline M & 57 & Ischemic & Right & 69 & 35 \\
\hline $\mathrm{F}$ & 61 & Ischemic & Left & 102 & 85 \\
\hline $\mathrm{F}$ & 65 & Haemorrhagic & Right & 94 & 100 \\
\hline M & 80 & Ischemic & Left & 72 & 70 \\
\hline M & 64 & Haemorrhagic & Right & 80 & 100 \\
\hline $\mathrm{F}$ & 73 & Haemorrhagic & Right & 80 & 35 \\
\hline M & 66 & Ischemic & Left & 62 & 100 \\
\hline M & 45 & Haemorrhagic & Right & 93 & 70 \\
\hline M & 74 & Ischemic & Bilateral & 101 & 100 \\
\hline
\end{tabular}




\begin{tabular}{clllll}
\hline F & 51 & Ischemic & Left & 112 & 100 \\
F & 63 & Ischemic & Left & 75 & 100 \\
F & 37 & Ischemic & Bilateral & 79 & 100 \\
F & 52 & Haemorrhagic & Left & 84 & 75 \\
F & 67 & Ischemic & Right & 81 & 55 \\
F & 85 & Ischemic & Right & 80 & 85 \\
F & 66 & Ischemic & Left & 79 & 20 \\
F & 79 & Haemorrhagic & Right & 76 & 85 \\
M & 76 & Ischemic & Right & 82 & 100 \\
M & 52 & Ischemic & Right & 80 & 45 \\
M & 72 & Ischemic & Right & 90 & 90 \\
F & 78 & Haemorrhagic & Right & 72 & 100 \\
F & 81 & Ischemic & Right & 124 & 100 \\
\hline $\mathrm{M}=52.5 \%$ & 65.55 & I = 68\% & R=50\% & 83.90 & 84.00 \\
$\mathrm{~F}=47.5 \%$ & $(14.13)$ & H = 32\% & L=35\% & $(15.65)$ & $(23.04)$ \\
& & & B =15\% & & \\
\hline
\end{tabular}

Notes: MBI - Modified Barthel Index; RBANS - Repeatable Battery for the Assessment of Neuropsychological Status. 
Table 2

Cronbach's Alpha Coefficients for the Personality Domains of the NEO-FFI for Stroke Participants, Informants for Stroke Participants and the Control Group (95\% Confidence Intervals $[\mathrm{CI}])$

\begin{tabular}{llllll}
\hline Group & $\mathrm{N}$ & $\mathrm{E}$ & $\mathrm{O}$ & $\mathrm{A}$ & $\mathrm{C}$ \\
\hline Stroke & .85 & .86 & .69 & .57 & .73 \\
\multicolumn{1}{c}{ CI } & $.77 \sim .91$ & $.79 \sim .92$ & $.52 \sim .81$ & $.34 \sim .74$ & $.58 \sim .84$ \\
Informants & .85 & .86 & .66 & .75 & .87 \\
\multicolumn{1}{c}{ CI } & $.74 \sim .93$ & $.76 \sim .93$ & $.41 \sim .85$ & $.56 \sim .88$ & $.77 \sim .94$ \\
Control & .83 & .73 & .75 & .81 & .68 \\
\multicolumn{1}{c}{ CI } & $.73 \sim .90$ & $.59 \sim .84$ & $.61 \sim .85$ & $.71 \sim .89$ & $.51 \sim .81$ \\
\hline
\end{tabular}

Note. $<.70=$ poor, $.7-.79=$ fair, $.8-.89=$ good, $\geq .90=$ excellent $($ Tate, 2010$)$ 
Table 3

Concordance between Self-Report (stroke participants) and Informant Reports on the NEOFFI at Time of Discharge

\begin{tabular}{lllll}
\hline Personality & Self-report & Informant & Intraclass & ICC Confidence \\
Domain & $M(S D)$ range & $M(S D)$ range & Coefficient & Interval (95\%) \\
& $(n=22)$ & $(n=22)$ & $($ ICC $)$ & \\
\hline Neuroticism & $49.27(12.20)$ & $47.60(10.52)$ & $.78^{* * *}$ & $.46 \sim .91$ \\
Extraversion & $25-69$ & $25-64$ & & $.24 \sim .87$ \\
Openness & $52.45(14.85)$ & $50.09(13.44)$ & $.68^{* *}$ & $.15 \sim .85$ \\
& $25-75$ & $25-75$ & & \\
Agreeableness & $47.09(9.99)$ & $44.75(9.60)$ & $.64^{*}$ & $.09 \sim .83$ \\
& $28-64$ & $28-69$ & & $.07 \sim .84$ \\
& $49.64(8.19)$ & $50.59(10.21)$ & $.58^{*}$ & \\
& $36-63$ & $30-67$ & & \\
\hline
\end{tabular}

Note. Intraclass coefficients $<.40=$ poor, $.4-.59=$ fair, .6-.74 = good, $\geq .75=$ excellent (Tate, 2010); ${ }^{*} p<.05, * * p<.01, * * * p<.001$ 
Table 4

Descriptive data on the NEO-FFI for the Control Group Comparison (time of discharge) and Longitudinal Stroke Sample

\begin{tabular}{|c|c|c|c|c|c|c|c|c|}
\hline \multirow{3}{*}{$\begin{array}{c}\text { NEO- } \\
\text { FFI }\end{array}$} & \multicolumn{3}{|c|}{ Stroke $(n=40)$ and Control Group $(n=40)$} & \multicolumn{3}{|c|}{ Longitudinal Stroke Sample $(n=27)$} & \multirow[b]{3}{*}{$F$} & \multirow[b]{3}{*}{$\eta_{p}^{2}$} \\
\hline & Stroke & Controls & & Discharge & 1-month & 4-months & & \\
\hline & $M(S D)$ & $M(S D)$ & $t(C I)$ & $M(S D)$ & $M(S D)$ & $M(S D)$ & & \\
\hline $\mathrm{N}$ & $50.25(11)$ & $48.70(10.91)$ & $-0.63(-6.41 \sim 3.33)$ & $49.15(11.56)$ & $49.56(12.68)$ & $49.52(13.41)$ & 0.05 & .004 \\
\hline $\mathrm{E}$ & $50.75(13.91)$ & $53.20(10.50)$ & $0.89(-3.04 \sim 7.94)$ & $49.00(13.25)^{\mathrm{ab}}$ & $44.56(12.05)^{\mathrm{a}}$ & $43.63(11.43)^{b}$ & $4.37 *$ & .259 \\
\hline $\mathrm{O}$ & $45.85(9.34)$ & 49.48 (11.39) & $1.56(-1.01 \sim 8.26)$ & $43.52(8.05)$ & $44.85(9.60)$ & $44.70(9.71)$ & 0.63 & .048 \\
\hline A & $46.43(9.39)$ & $48.50(13.62)$ & $0.79(-3.14 \sim 7.28)$ & $47.26(9.81)^{\mathrm{ab}}$ & $50.74(8.42)^{\mathrm{a}}$ & $50.93(9.33)^{b}$ & $4.29 *$ & .255 \\
\hline $\mathrm{C}$ & 50.65 (9.13) & $50.58(8.48)$ & $-0.38(-4.00 \sim 3.85)$ & $51.30(9.42)$ & $51.96(7.50)$ & $49.56(8.58)$ & 1.13 & .083 \\
\hline
\end{tabular}

Note. ${ }^{*} \mathrm{p}<.05,{ }^{* *} \mathrm{p}<.01,{ }^{* * *} \mathrm{p}<.001 ;$ Note: Significant findings for post-hoc tests with Bonferroni adjustment $(p<.017)$ are denoted with superscript 
Table 5

Pearson Product-Moment Correlations between Self-rated Personality Domains and Mood for Stroke Participants at Time of Discharge $(n=40)$

\begin{tabular}{|c|c|c|c|c|c|}
\hline & $\mathrm{N}$ & $\mathrm{E}$ & $\mathrm{O}$ & A & $\mathrm{C}$ \\
\hline Neuroticism & - & & & & \\
\hline Extraversion & $-.50 * *$ & & & & \\
\hline Openness & -.12 & .29 & & & \\
\hline Agreeableness & $-.70 * * *$ & $.40^{*}$ & .10 & & \\
\hline Conscientiousness & .04 & .12 & -.26 & .25 & \\
\hline Depression (CES-D) & $.50 * *$ & $-.36 *$ & .17 & -.20 & .20 \\
\hline Anxiety (GAI) & $.68^{* * *}$ & $-.33 *$ & .11 & $-.43 * *$ & .17 \\
\hline
\end{tabular}

